Research Square

\title{
What Factors Contribute to Moral Problems in Patient Care? A Scoping Review and Case Series of Ethics Consultations at a Somatic and a Psychiatric University Hospital in Switzerland
}

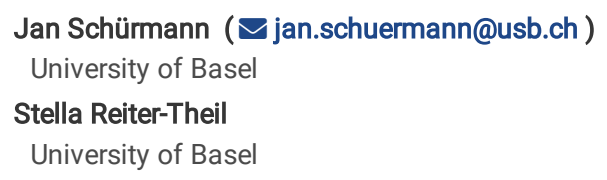

\section{Research Article}

Keywords: preventive ethics, clinical ethics, ethics support, ethics consultation, prevention, risk factor, moral conflict, psychiatry, case series, scoping review

Posted Date: February 22nd, 2022

DOI: https://doi.org/10.21203/rs.3.rs-1294882/v1

License: (c) (i) This work is licensed under a Creative Commons Attribution 4.0 International License. Read Full License 


\section{Abstract \\ Background}

Health care professionals (HCPs) are regularly exposed to moral problems arising in patient care that may compromise quality of care. An understanding of risk factors and early indicators may help practitioners to address these problems early on. This study aims to provide an overview of moral risk factors in patient care. Risk factors known from the literature and those that have led to moral problems discussed in clinical ethics consultations (CECs) are examined. Characteristics, ethical issues and outcomes of CEC are also presented.

\section{Methods}

Scoping review on moral risk factors in patient care; CEC case series with semi-structured analysis of documentation (consultation reports, feedback forms, electronic patient records) from 204 CECs held between 2012 and 2020 at a somatic (USB) and a psychiatric (UPK) university hospital in Basel, Switzerland.

\section{Results}

Ninety-nine moral risk factors were identified from the literature referring to four risk areas: patient (41), family (12), health care professional (29) and system (17). Eighty-seven of these were documented at least once in CEC. The most prevalent factors were patient vulnerability (100\%), absent or unclear hospital policies (97.1\%), shift work (83.3\%), inadequate understanding between patient and HCPs (73.5\%), inadequate communication (66.2\%), disagreement between patient and HCPs about care (58.8\%), and multiple care teams (53.4\%). The prevalence differs significantly depending on the medical specialty. CECs are requested as often in psychiatry as in somatic medicine. In the USB, ethical questions about life-sustaining treatment (27.8\%), maternal-foetal conflicts (18.8\%) and assisted reproductive technology (10.5\%) are the most prevalent; in the UPK, questions about coercive measures (41.8\%), risk and opportunity assessment (14.9\%) and child welfare endangerment (13.4\%) predominate. Overall, a consensus was almost always reached among the participants (98.5\%) and the results implemented (93.9\%). CECs were rated as helpful (95.0\%) or partly helpful (5.0\%).

\section{Conclusions}

CEC is useful in both somatic medicine and psychiatry to find consensual and helpful solutions to moral conflicts. There are highly prevalent and potent moral risk factors in all dimensions relevant in clinical care that can be used to prevent moral problems. Further empirical research is needed to create different risk profiles specific to the medical specialities.

\section{Introduction}

Clinical ethics consultation (CEC) is a well-established form of clinical ethics support (CES). In the US, $86 \%$ of all hospitals have a clinical ethics support service (CESS); 74\% actively provide CEC (1). CEC is also increasingly implemented in hospitals in European countries such as Germany, the UK and Switzerland (2-4). To increase prevalence and quality of CEC, recommendations by medical associations $(5,6)$, professional standards $(7-9)$, training guides and courses (10-12), certification and accreditation programmes (13-16) and tools to evaluate CEC (17-19) have been developed. There is also now some evidence for positive outcomes of CEC regarding family and healthcare professional (HCP) satisfaction, assistance with ethical decision-making, perceived impact on clinical care, moral competency and quality of communication (20-22).

Not as well established or studied are forms of preventive clinical ethics support (PCES). Unlike classic CEC, which is initiated in response to a request from stakeholders, PCES engages proactively with the aim of preventing the occurrence, continuation, or intensification of moral uncertainty, conflicts or dilemma (in short: moral problems) in patient care. Since the first conception of "preventive ethics" in the 1990s $(23,24)$, different approaches with various proactive interventions have been proposed, including a CES needs assessment survey (25), ethics screening (26, 27), ethics rounds (28), regular ethical case discussions (29), proactive ethics consultation $(30,31)$, decision aids (32) and process assessment (33). PCES assumes that moral problems in clinical care often develop gradually over time and intensify if there is no early ethical intervention. A process model distinguishing different phases of moral problems and primary, secondary and tertiary prevention has been proposed (34).

To be effective, prevention needs to be targeted at specific risk factors or early indicators. Moral risk factors can be defined as features of a situation that increase the chance of moral problems arising; early indicators show early on that a moral problem is arising. Although there are individual studies on moral risk factors in patient care, there currently is no systematic overview of highly prevalent or potent risk factors in general or related to different medical specialities. This study aims to provide such an overview based on the current state of research.

So far, CECs have rarely been used to examine moral risk factors. Some moral conflicts in patient care, presumably those that are particularly complex or burdensome, are addressed in CEC. Each situation dealt with in CEC has a risk profile that triggered one or more moral problems in that individual case. CECs are therefore suitable situations through which to study risk factors for full-blown moral problems in clinical practice. This study uses a case series to screen CECs for moral risk factors. Case series have been widely used to assess ethical issues, users, case characteristics and outcomes of CEC (35-44). As case series cannot use a control group, the relative risk for moral conflict cannot be statistically calculated. However, risk factors with a high prevalence, factors with a presumably lower prevalence in the overall population or factors previously identified in the literature may provide clues to their own relevance (45). This study aims to contribute to the development of specific, prevalent and potent moral risk factors that can be used to prevent moral conflicts in patient 
care. At the same time, the study sheds light on the demographic, clinical and formal characteristics; ethical issues; and outcomes of CEC provided by our service.

\section{Methods}

\section{Research setting}

The Clinical Ethics Unit Basel in Switzerland is responsible for providing CES at the University Hospital Basel (USB), a tertiary somatic hospital with 800 beds (without paediatric and psychiatric wards), and the University Psychiatric Clinics Basel (UPK), a psychiatric hospital with 300 beds, both with in- and outpatient settings. The Basel approach is oriented towards "ethics facilitation", conceptualising CEC as a facilitated discussion to support HCPs, patients or relatives with moral problems in patient care $(8,40)$. All stakeholders can request ethics support. CEC is usually attended by two trained ethics consultants (who moderate and take notes), attending physicians and nurses; occasionally, patients, relatives, legal guardians, therapists and social workers also take part. The focus of the discussion is on identifying, analysing and jointly evaluating moral problems and options with the aim of developing an ethically justified and consensual procedure. While the ethics consultants are responsible for the process and the quality of ethical justification, the responsibility for decisionmaking and acting remains with the clinicians and patients, respectively (46). Each CEC is documented by the ethics consultants in a report, which is filed in the electronic patient record, and a standardised feedback form is obtained from the requesting person. In addition to CEC, further ethics support is provided; this may include ethics rounds, team-internal ethical case discussions, ethics training or ethics policy development (47).

\section{Research design}

This study aims to provide an overview of moral risk factors in patient care. In addition, a comparison is made between somatic and psychiatric CECs regarding their characteristics, ethical issues and outcomes. For these purposes, a two-step procedure is used: First, a scoping review examines the evidence in the literature on moral risk factors, including a previous expert interview study by the authors (34). This is followed by a CEC case series in which CEC documentation is analysed by operationalising the identified risk factors and using codes predefined by our CES quality assessment.

\section{Scoping review}

The review follows the PRISMA extension for scoping reviews (48). A review protocol was prepared in advance. All types of publications between 1970 and 2021 in English, German and French were considered as sources of evidence. The databases PubMed, Scopus, Web of Science, Google Scholar and BELIT were searched on 27/08/2021. A keyword search algorithm was used that combines the terms "risk factor", "ethical issue" and "patient care".[1] For further evaluation, publications were selected that, according to the abstract and subsequently the main text, examine moral risk factors in patient care. Further literature was obtained by the snowball method of reviewing the literature referenced in the included articles.

A data-charting form was developed, discussed and continuously updated using IBM SPSS Statistics 27. The moral risk factors found were compared, pooled, systematised and ranked. The ranking was made by analysing the frequency with which the risk factors were identified in the studies using Microsoft Excel 356 (only studies with statistical analysis were considered).

\section{Case series}

The retrospective, consecutive case series includes all case-related CECs performed and documented at the USB and UPK between August 2012 and April 2020 ( $n=204$ ); it follows the criteria of the JBI critical appraisal checklist for case series (49). Cases not sufficiently documented (according to the standardised in-house template for CEC reports) $(n=12)$, as well as CECs without reference to an individual case $(n=9)$, were excluded. Other forms of ethics support were not considered.

Standardised CEC reports, feedback forms and electronic patient records were analysed by structured content analysis (50). The highly structured CEC reports contains information on, amongst other things, the request, participants, duration, medical and social situation, patient preferences and quality of life, decisional capacity, advance directive and substitute decision-maker, guidelines and laws, main and secondary ethical issues, options, ethical analysis, conclusion, and consensus achieved in the CECs. The analysis draws on a CEC database in which the authors have recorded this information from the reports, using IBM SPSS Statistics. All reports $(n=204)$ were analysed and coded again to ensure coding reliability. The database categories had been previously checked, revised and extended by the authors. In particular, the risk factors identified in the literature review were operationalised and added to the code set. All items of the feedback forms, including questions on further clinical course, usefulness of CEC and documentation, response time and comments, were also included in the analysis $(n=143$, response rate $70.1 \%)$. All electronic patient records available for the CEC cases $(n=177)$ were coded according to previously defined codes, including nationality, survival rate, surrogacy and other codes related to the identified risk factors. No patient files were available for some cases $(n=27)$ due to the anonymisation of reports and patients from external institutions. All data were anonymised and encrypted before analysis. Data was analysed by descriptive statistics using IBM SPSS Statistics 28.

\section{Footnote:}

[1] Search string for PubMed: ((risk factor[MeSH Terms]) AND (ethical issue[MeSH Terms]) AND (patient care[MeSH Terms]))

\section{Results}

\section{Results from the scoping review on moral risk factors}


A total of 664 records were found in the search, 541 without duplicates. Thirty-three records were excluded due to missing information. 503 records were excluded for lack of relevance, and five records were assessed as eligible. Through the snowball method, a further four eligible publications were found; accordingly, nine studies were included in the synthesis (see Fig. 1).

The results of the studies included are shown in Table 1. All but one of the studies come from the US; five focus on intensive care. Methodologically, the studies are very heterogeneous, ranging from expert panels, case studies, surveys and interview studies to validation studies for screening tools. Most of the studies carry out validation using descriptive statistics. Only Robinson et al. (2017) use a retrospective control group and analyse the data using inferential statistics. Pavlish et al. validate their results in later studies by evaluating the implementation of an Ethics Early Action Protocol (26, 51, 52).

Table 1

Results of individual sources of evidence on moral risk factors

\begin{tabular}{|c|c|c|c|c|c|c|c|c|c|}
\hline Authors & Year & Origin & $\begin{array}{l}\text { Medical } \\
\text { specialty }\end{array}$ & Sample & $\begin{array}{l}\text { Sample } \\
\text { size }\end{array}$ & Methods & $\begin{array}{l}\text { Statistical } \\
\text { analysis }\end{array}$ & $\begin{array}{l}\text { Number } \\
\text { of risk } \\
\text { factors }\end{array}$ & Risk areas cited \\
\hline $\begin{array}{l}\text { Anderson- } \\
\text { Shaw et al. } \\
\text { (53) }\end{array}$ & 2007 & USA & $\begin{array}{l}\text { Emergency } \\
\text { care }\end{array}$ & Patients & 50 & $\begin{array}{l}\text { Analysis } \\
\text { of } \\
\text { electronic } \\
\text { patient } \\
\text { records for } \\
\text { patients } \\
\text { with CEC } \\
\text { in / not in } \\
\text { emergency } \\
\text { care }\end{array}$ & $\begin{array}{l}\text { Yes } \\
\text { (descriptive } \\
\text { statistics) }\end{array}$ & 5 & None \\
\hline Epstein (54) & 2012 & USA & $\begin{array}{l}\text { Intensive } \\
\text { care }\end{array}$ & Patients & 1 & $\begin{array}{l}\text { Case } \\
\text { study; } \\
\text { literature } \\
\text { search }\end{array}$ & No & 21 & $\begin{array}{l}\text { Patient/relatives/surrogates- } \\
\text { level risk factors, Unit-level risk } \\
\text { factors, System-level risk } \\
\text { factors }\end{array}$ \\
\hline Heyl (55) & 2008 & USA & Mixed & $\begin{array}{l}\text { Clinical } \\
\text { ethics } \\
\text { committee } \\
\text { members }\end{array}$ & $\begin{array}{l}\text { not } \\
\text { available }\end{array}$ & $\begin{array}{l}\text { Risk } \\
\text { factors } \\
\text { defined by } \\
\text { clinical } \\
\text { ethics } \\
\text { committee }\end{array}$ & No & 3 & None \\
\hline $\begin{array}{l}\text { Morgenstern } \\
(56)\end{array}$ & 2005 & USA & $\begin{array}{l}\text { Intensive } \\
\text { care }\end{array}$ & $\begin{array}{l}\text { Clinical } \\
\text { ethics } \\
\text { committee } \\
\text { members }\end{array}$ & 45 & $\begin{array}{l}\text { Risk } \\
\text { factors } \\
\text { defined by } \\
\text { clinical } \\
\text { ethics } \\
\text { committee }\end{array}$ & No & 12 & None \\
\hline $\begin{array}{l}\text { Pavlish et } \\
\text { al. (57) }\end{array}$ & 2011 & USA & $\begin{array}{l}\text { Intensive } \\
\text { care }\end{array}$ & Nurses & 70 & $\begin{array}{l}\text { Survey } \\
\text { (critical } \\
\text { incident } \\
\text { technique) }\end{array}$ & $\begin{array}{l}\text { Yes } \\
\text { (descriptive } \\
\text { statistics) }\end{array}$ & 36 & $\begin{array}{l}\text { Individual risk factors, Family } \\
\text { risk factors, Health care } \\
\text { professional risk factors, } \\
\text { System/organisational/society } \\
\text { risk factors, Multilevel risk } \\
\text { factors }\end{array}$ \\
\hline $\begin{array}{l}\text { Pavlish et } \\
\text { al. (52) }\end{array}$ & $2015 a$ & USA & $\begin{array}{l}\text { Intensive } \\
\text { care }\end{array}$ & Patients & 55 & $\begin{array}{l}\text { Screening } \\
\text { instrument }\end{array}$ & $\begin{array}{l}\text { Yes } \\
\text { (descriptive } \\
\text { statistics) }\end{array}$ & 21 & $\begin{array}{l}\text { Individual risk factors, Family } \\
\text { risk factors, Health care team } \\
\text { risk factors, Health care } \\
\text { system risk factors, Multilevel } \\
\text { risk factors }\end{array}$ \\
\hline $\begin{array}{l}\text { Pavlish et } \\
\text { al. (58) }\end{array}$ & $2015 c$ & USA & Mixed & Physicians & 114 & Survey & $\begin{array}{l}\text { Yes } \\
\text { (descriptive } \\
\text { statistics) }\end{array}$ & 25 & $\begin{array}{l}\text { Interactional circumstances, } \\
\text { Personal circumstances, } \\
\text { System circumstances }\end{array}$ \\
\hline $\begin{array}{l}\text { Robinson et } \\
\text { al. (41) }\end{array}$ & 2017 & USA & $\begin{array}{l}\text { Intensive } \\
\text { care }\end{array}$ & Patients & 310 & $\begin{array}{l}\text { Analysis } \\
\text { of } \\
\text { electronic } \\
\text { patient } \\
\text { records } \\
\text { and CEC } \\
\text { records }\end{array}$ & $\begin{array}{l}\text { Yes } \\
\text { (inferential } \\
\text { statistics) }\end{array}$ & 5 & None \\
\hline $\begin{array}{l}\text { Schürmann } \\
\text { et al. (34) }\end{array}$ & 2021 & Switzerland & Mixed & $\begin{array}{l}\text { Health care } \\
\text { professionals }\end{array}$ & 20 & $\begin{array}{l}\text { Expert } \\
\text { interview } \\
\text { and survey }\end{array}$ & $\begin{array}{l}\text { Yes } \\
\text { (descriptive } \\
\text { statistics) }\end{array}$ & 60 & $\begin{array}{l}\text { Patient risk factors, Family risk } \\
\text { factors, Health care } \\
\text { professional risk factors, } \\
\text { Systemic risk factors }\end{array}$ \\
\hline
\end{tabular}

CEC: clinical ethics consultation.

Ninety-nine moral risk factors were identified, which can be assigned to four risk areas: patient (41), family (12), health care professional (29) and system (17). The grouped and ranked risk factors are shown in Table 2. The top 20 ranked risk factors are (ordered by rank, without subcategories): patient vulnerability, end-of-life situation, inadequate communication, incoherent treatment plan, different values and world views, inadequate resources, inadequate moral attitude 
of HCPs, inadequate understanding of disease on the part of relatives, unnecessary suffering of the patient, lack of crucial information or evidence, disagreement among HCPs about care, overworked HCPs, disagreement between relatives and HCPs about care, disagreement among relatives about care, fear and risk behaviour (defensive medicine), unclear treatment plan among relatives, unclear or missing preferences or advance directive of the patient, challenging clinical situations, inadequate institutional framework and poor ethical climate in the team. 


\section{Risk factors*}

\section{A. Risk area: patient}

Vulnerability (1) $2,5,6,9$

- lack of decisional capacity (6) $1,2,5,7,8,9 \quad$ - foreign language (43) $2,5,8,9$

- very young or old age (9) $2,4,5,7,8,9$

- alone (24) $2,5,6,7$

- mental illness (31) $5,7,9$

- state of neglect (31) $5,7,9$

- drug dependence (39) 7,9

- lack of education (39) 7,9

- multimorbidity (40) $1,4,7,8$

End-of-life situation (2) $2,4,5,6,8,9$

Unnecessary suffering (11) 5,6

Unclear or missing preferences or advance directive (19) ${ }^{1,8,9}$

Challenging clinical situations (21) ${ }^{9}$

- medical limits of treatment (11) $5,6,9$

- maternal-foetal conflict (69) ${ }^{9}$

- involuntary treatment (69) ${ }^{9}$

- isolated patient (69) ${ }^{9}$

Inadequate understanding between patient and HCP (24) ${ }^{9}$

Prolonged intensive therapy (34) 4,9

Overburdened patient (34) ${ }^{9}$

Self-harm and harm to others (34) ${ }^{9}$

Disagreement about care between patient and $\operatorname{HCP}(45)^{2,3,9}$

Inadequate understanding of disease (patient) (45) 2,9

Imminently dying (45) ${ }^{6}$

Acute renal failure (69) ${ }^{4}$

Unclear or missing CPR code (69) ${ }^{8}$

Intractable pain (69) ${ }^{4}$

Multiple decubitus (69) ${ }^{4}$

Multiple re-admissions (69) ${ }^{4}$

Profound neurological deficit (69) ${ }^{4}$

Profound paralysis (69) ${ }^{4}$

Prolonged artificial respiration (69) ${ }^{4}$

Recurrent aspiration pneumonia (69) ${ }^{4}$

Repeated life-sustaining treatments (69) ${ }^{8}$

\section{Risk area: health care professional}

Incoherent treatment plan (4) 2,3,5,6,7,9

Inadequate moral attitude (8) 5,9

- hubris (69) ${ }^{9}$

- lack of moral commitment (69) ${ }^{9}$

- maturity lacking (69) ${ }^{9}$

- prejudice against patients (69) ${ }^{9}$

- lack of moral sensitivity (HCP) (69) ${ }^{9}$

Disagreement about care among HCP (14) 2,5,6,9

Overworked HCP (14) $)^{7,9}$

Fear and risk behaviour (defensive medicine) (18) 5,9

Poor ethical climate in the team (21) ${ }^{9}$

- lack of discussion culture (69) ${ }^{9} \quad$ - climate of fear (69) ${ }^{9}$

- lack of moral sensitivity (Team) (69) ${ }^{9}$ - no common attitude (69) ${ }^{9}$

Strong team hierarchy (24) ${ }^{9}$

Treatment standards not considered adequately (29) 5,6

Avoidance of end-of-life or difficult discussions (33) 2,5,6

Role ambiguity (34) ${ }^{9}$

Inadequate skills or knowledge (45) ${ }^{9}$

Insufficient understanding among $\mathrm{HCP}(45)^{9}$

Emotionally involved (52) ${ }^{7}$

Overly aggressive $(52)^{7}$

Disagreement about evidence among $\operatorname{HCP}(55)^{6}$

Inadequate understanding of disease (HCP) (64) ${ }^{5}$

Uncertainty about decisional capacity (66) ${ }^{6}$

Frequent HCP turnover (69) ${ }^{2}$

Late or absent family meetings (69) ${ }^{2}$

Ineffective handover (69) ${ }^{2}$

\section{Risk area: system}

Inadequate communication (3) 5,6,7,9

Different values or world views (5) $2,5,7,9$

Inadequate resources (7) $2,5,7,9$

Lack of crucial information or evidence (11) 5,9

Inadequate institutional framework (21) ${ }^{9}$

* Ordered by rank (in brackets) within the groups.

${ }^{1}$ Anderson-Shaw et al. (2007); ${ }^{2}$ Epstein (2012); ${ }^{3}$ Heyl (2008); ${ }^{4}$ Morgenstern (2005); ${ }^{5}$ Pavlish et al. (2011); ${ }^{6}$ Pavlish et al. (2015a); ${ }^{7}$ Pavlish et al. (2015c);

${ }^{8}$ Robinson et al. (2017); ${ }^{9}$ Schürmann et al. (2021). 


\begin{tabular}{|c|c|c|}
\hline \multicolumn{3}{|l|}{ Risk factors* } \\
\hline & - lack of management support (52) 7,9 & - ongoing reorganisation (69) 9 \\
\hline B. Risk area: family & - routines impeding care (69) 2,9 & - shift work (69) ${ }^{9}$ \\
\hline Inadequate understanding of disease (relatives) (9) 2,5,6,9 & \multicolumn{2}{|l|}{ Wrong incentives $(24)^{9}$} \\
\hline Disagreement about care between relatives $\& \operatorname{HCP}(16)^{2,3,5,6,9}$ & \multicolumn{2}{|c|}{ System promotes non-beneficial care (29) ${ }^{5}$} \\
\hline Disagreement about care among relatives (17) $5,6,9$ & \multicolumn{2}{|l|}{ Public pressure (34) ${ }^{9}$} \\
\hline Unclear treatment plan among relatives (18) 5,6 & \multicolumn{2}{|l|}{ Inadequate legislation or standards (45) ${ }^{9}$} \\
\hline Substitute function not fulfilled appropriately (24) 5,9 & \multicolumn{2}{|c|}{ Social developments or medical progress (45) ${ }^{9}$} \\
\hline Infrequent or missing visits by relatives (42) $2,5,6$ & \multicolumn{2}{|l|}{ Absent or unclear hospital policies (55) 2,6} \\
\hline Lack of education among relatives $(58)^{7}$ & \multicolumn{2}{|l|}{ Strong institutional hierarchy (57) ${ }^{5}$} \\
\hline Low trust in $\mathrm{HCP}(58)^{7}$ & \multicolumn{2}{|l|}{ Multiple care teams (58) ${ }^{5}$} \\
\hline \multicolumn{3}{|l|}{ Negative experiences (58) ${ }^{7}$} \\
\hline \multicolumn{3}{|l|}{ Multiple family members $(58)^{7}$} \\
\hline \multicolumn{3}{|l|}{ Poor decision-making skills $(58)^{7}$} \\
\hline \multicolumn{3}{|l|}{ Substitute missing or not accessible (69) ${ }^{1}$} \\
\hline \multicolumn{3}{|l|}{ * Ordered by rank (in brackets) within the groups. } \\
\hline \multicolumn{3}{|c|}{$\begin{array}{l}1 \text { Anderson-Shaw et al. (2007); }{ }^{2} \text { Epstein (2012); }{ }^{3} \mathrm{Heyl} \text { (2008); }{ }^{4} \text { Morgenstern (2005); }{ }^{5} \text { Pavlish et al. (2011); }{ }^{6} \text { Pavlish et al. (2015a); }{ }^{7} \text { Pavlish et al. (2015c) } \\
8 \text { Robinson et al. (2017); }{ }^{9} \text { Schürmann et al. (2021). }\end{array}$} \\
\hline
\end{tabular}

HCP: health care professionals.

\section{Results from the CEC case series}

Formal characteristics of CECs are shown in Table 3. CECs were mostly prospective (82.4\%) covering a wide range of clinical fields. The requests came most frequently from the women's health clinic (21.6\%), adult psychiatry (20.6\%) and internal medicine (15.2\%). In almost two thirds of all cases, the request for CEC was made by physicians and in almost a quarter by the nursing staff, nearly exclusively by head nurses or nursing experts. In the UPK, one fifth of the requests came from therapists, that is psychologists or social workers. Patients and relatives rarely requested a CEC (2.9\%) or attended a CEC in person (9.8\%). On average, a CEC had 10 [4.3] participants and lasted 69 [20-135] minutes. In the USB, almost half of the requests were urgent or very urgent, meaning consultation was requested within 48 or 24 hours, respectively. 
Table 3

Formal characteristics of CEC

\begin{tabular}{|c|c|c|c|c|c|}
\hline & & \multicolumn{3}{|l|}{ Hospital } & \multirow[t]{2}{*}{ Total } \\
\hline & & USB & UPK & External & \\
\hline & & $\mathrm{n}=133(\%)$ & $\mathrm{n}=67(\%)$ & $\mathrm{n}=4(\%)$ & $\mathrm{n}=204(\%)$ \\
\hline \multirow[t]{2}{*}{ Form of CEC } & Prospective CEC & $116(87.2)$ & $48(71.6)$ & $4(100.0)$ & $168(82.4)$ \\
\hline & Retrospective CEC & $17(12.8)$ & $19(28.4)$ & $0(0.0)$ & $36(17.6)$ \\
\hline \multirow{11}{*}{$\begin{array}{l}\text { Requesting } \\
\text { department }\end{array}$} & Women's Health Clinic & $44(33.1)$ & $0(0.0)$ & $0(0.0)$ & $44(21.6)$ \\
\hline & Adult Psychiatry & $0(0.0)$ & $42(62.7)$ & $0(0.0)$ & $42(20.6)$ \\
\hline & Internal Medicine & $31(23.3)$ & $0(0.0)$ & $0(0.0)$ & $31(15.2)$ \\
\hline & Surgery & $26(19.5)$ & $0(0.0)$ & $0(0.0)$ & $26(12.7)$ \\
\hline & Forensic Psychiatry & $0(0.0)$ & $13(19.4)$ & $0(0.0)$ & $13(6.4)$ \\
\hline & Child and Adolescent Psychiatry & $0(0.0)$ & $11(16.4)$ & $0(0.0)$ & $11(5.4)$ \\
\hline & Intensive Care Unit (MICU/SICU) & $10(7.5)$ & $0(0.0)$ & $0(0.0)$ & $10(4.9)$ \\
\hline & Neurology or Neurosurgery & $5(3.8)$ & $0(0.0)$ & $0(0.0)$ & $5(2.5)$ \\
\hline & Acute Geriatrics & $5(3.8)$ & $0(0.0)$ & $0(0.0)$ & $5(2.5)$ \\
\hline & Emergency Department & $4(3.0)$ & $0(0.0)$ & $0(0.0)$ & $4(1.9)$ \\
\hline & Other & $8(6.0)$ & $1(1.5)$ & $4(100.0)$ & $13(6.4)$ \\
\hline \multirow{6}{*}{$\begin{array}{l}\text { Requesting } \\
\text { profession }\end{array}$} & Physician & $91(68.4)$ & $32(47.8)$ & $3(75.0)$ & $126(61.8)$ \\
\hline & Nurse & $33(24.8)$ & $14(20.9)$ & $0(0.0)$ & $47(23.0)$ \\
\hline & Therapist & $0(0.0)$ & $14(20.9)$ & $1(25.0)$ & $15(7.4)$ \\
\hline & Patient or Relative & $4(3.0)$ & $2(3.0)$ & $0(0.0)$ & $6(2.9)$ \\
\hline & Social worker & $1(0.8)$ & $1(1.5)$ & $0(0.0)$ & $2(1.0)$ \\
\hline & Other & $4(3.0)$ & $4(6.0)$ & $0(0.0)$ & $8(3.9)$ \\
\hline \multicolumn{2}{|c|}{ Number of participants [M (SD)] } & $10.0[4.0]$ & $10.3[5.0]$ & $5.3[2.2]$ & $10.0[4.3]$ \\
\hline \multicolumn{2}{|c|}{ Duration [min (Range)] } & $64 \min [20-105]$ & $77 \min [30-135]$ & $84 \min [60-130]$ & $69 \min [20-135]$ \\
\hline \multirow[t]{3}{*}{ Urgency } & normal & $70(52.6)$ & $67(100.0)$ & $4(100.0)$ & $141(69.1)$ \\
\hline & urgent & $25(18.8)$ & $0(0.0)$ & $0(0.0)$ & 25 (12.3) \\
\hline & very urgent & $38(28.6)$ & $0(0.0)$ & $0(0.0)$ & 38 (18.6) \\
\hline
\end{tabular}

Demographic and clinical characteristics of the patients whose cases were discussed in CEC are shown in Table 4. There is a slight preponderance of female patients (58.3\%). The mean age is 48.7 years, with three quarters being between 19 and 69 years old. On average, patients from the UPK are more than ten years younger than patients from the USB. Two thirds of the patients are Swiss, and only one in ten is non-European. Overall, the patients suffer most often from mental illness (32.4\%); most prevalent in USB patients are pregnancy-related disorders (18.8\%), malignant neoplasms (15.0\%) and neurological diseases (13.5\%). The prognosis regarding the main disease with treatment is poor for almost a third of all patients, intermediate for a quarter, and terminal for one in ten. Of the USB patients, almost one fifth died in hospital and almost one third within six months of discharge. Less than half of all patients had decisional capacity regarding treatment decisions, according to the assessment of the HCPs participating in the CEC. Patients lacked decisional capacity in $28.4 \%$ of all cases; in $8.3 \%$, capacity fluctuated. For two thirds of UPK patients, decisional capacity was either not given (29.9\%), fluctuating (7.5\%) or unclear (31.3\%). Only $12.8 \%$ of all patients had an advance directive; slightly more did so in the USB (17.4\%). Of all patients, $37.9 \%$ had a defined substitute decision-maker; in the UPK, almost half of the patients did. 
Table 4

Demographic and clinical characteristics of patients discussed in CEC

\begin{tabular}{|c|c|c|c|c|c|}
\hline & & \multicolumn{3}{|l|}{ Hospital } & \multirow[t]{2}{*}{ Total } \\
\hline & & USB & UPK & External & \\
\hline & & $\mathrm{n}=133(\%)$ & $\mathrm{n}=67(\%)$ & $\mathrm{n}=4(\%)$ & $\mathrm{n}=204(\%)$ \\
\hline \multirow[t]{2}{*}{ Sex } & Female & $80(60.2)$ & $38(56.7)$ & $1(25.0)$ & $119(58.3)$ \\
\hline & Male & $53(39.8)$ & $29(43.3)$ & $3(75.0)$ & 85 (41.7) \\
\hline \multirow[t]{6}{*}{ Age } & Mean [Range] & 52,4 y [10-93] & 39,9 y [0-90] & 68,8 y [52-84] & 48,7 y $[0-93]$ \\
\hline & $\leq 1$ year & $0(0.0)$ & $2(3.0)$ & $0(0.0)$ & $2(1.0)$ \\
\hline & $2-18$ years & $2(1.5)$ & $11(16.7)$ & $0(0.0)$ & $13(6.4)$ \\
\hline & $19-49$ years & $63(47.4)$ & $27(40.9)$ & $0(0.0)$ & $90(44.3)$ \\
\hline & $50-69$ years & $37(27.8)$ & $22(33.3)$ & $2(50.0)$ & $61(30.0)$ \\
\hline & $\geq 70$ years & $31(23.3)$ & $4(6.1)$ & $2(50.0)$ & $37(18.2)$ \\
\hline \multirow[t]{8}{*}{ Nationality } & Central Europe & $93(72.1)$ & $58(86.6)$ & $4(100.0)$ & $155(77.5)$ \\
\hline & - Switzerland & $75(58.1)$ & $56(83.6)$ & $4(100.0)$ & $135(67.5)$ \\
\hline & Western Europe & $5(3.9)$ & $0(0.0)$ & $0(0.0)$ & $5(2.5)$ \\
\hline & Eastern Europe & $2(1.6)$ & $0(0.0)$ & $0(0.0)$ & $2(1.0)$ \\
\hline & Southern Europe & $5(3.9)$ & $3(4.5)$ & $0(0.0)$ & $8(4.0)$ \\
\hline & Southeast Europe & $6(4.7)$ & $2(3.0)$ & $0(0.0)$ & $8(4.0)$ \\
\hline & Asia & $16(12.4)$ & $3(4.5)$ & $0(0.0)$ & $19(9.5)$ \\
\hline & Africa & $2(1.6)$ & $1(1.5)$ & $0(0.0)$ & $3(1.5)$ \\
\hline \multirow[t]{2}{*}{ Insurance status } & insured & $131(98.5)$ & $67(100.0)$ & $4(100.0)$ & $202(99.0)$ \\
\hline & uninsured & $2(1.5)$ & $0(0.0)$ & $0(0.0)$ & $2(1.0)$ \\
\hline \multirow[t]{13}{*}{ Main diagnosis } & Mental disorder & $8(6.0)$ & $56(83.6)$ & $2(50.0)$ & $66(32.4)$ \\
\hline & Pregnancy-related disorder ${ }^{1}$ & $25(18.8)$ & $0(0.0)$ & $0(0.0)$ & $25(12.3)$ \\
\hline & Malignant neoplasm & $20(15.0)$ & $0(0.0)$ & $2(50.0)$ & $22(10.8)$ \\
\hline & Neurological disease & $18(13.5)$ & $3(4.5)$ & $0(0.0)$ & $21(10.3)$ \\
\hline & Pulmonary disease & $12(9.0)$ & $0(0.0)$ & $0(0.0)$ & $12(5.9)$ \\
\hline & Cardiovascular disease & $10(7.5)$ & $2(3.0)$ & $0(0.0)$ & $12(5.9)$ \\
\hline & Metabolic disease & $5(3.8)$ & $4(6.0)$ & $0(0.0)$ & $9(4.4)$ \\
\hline & Genitourinary disease & $8(6.0)$ & $0(0.0)$ & $0(0.0)$ & $8(3.9)$ \\
\hline & Gastrointestinal disease & $6(4.5)$ & $0(0.0)$ & $0(0.0)$ & $6(2.9)$ \\
\hline & Congenital or genetic disease & $6(4.5)$ & $0(0.0)$ & $0(0.0)$ & $6(2.9)$ \\
\hline & Multiple organ dysfunction syndrome & $4(3.0)$ & $0(0.0)$ & $0(0.0)$ & $4(2.0)$ \\
\hline & Other & $9(6.8)$ & $1(1.5)$ & $0(0.0)$ & $10(4.9)$ \\
\hline & None & $2(1.5)$ & $1(1.5)$ & $0(0.0)$ & $3(1.5)$ \\
\hline \multirow[t]{5}{*}{ Prognosis } & good & $28(21.5)$ & $10(15.4)$ & $0(0.0)$ & 38 (19.2) \\
\hline & intermediate & $39(30.0)$ & $15(23.1)$ & $0(0.0)$ & $54(27.3)$ \\
\hline & poor & $40(30.8)$ & $23(35.4)$ & $2(66.7)$ & $65(32.8)$ \\
\hline & terminal & $17(13.1)$ & $3(4.6)$ & $0(0.0)$ & $20(10.1)$ \\
\hline & unclear & $6(4.6)$ & $14(21.5)$ & $1(33.3)$ & $21(10.6)$ \\
\hline \multirow[t]{2}{*}{ Survival } & survived up to discharge & $105(80.8)$ & $66(98.5)$ & $4(100.0)$ & $175(87.1)$ \\
\hline & died in hospital & $25(19.2)$ & $1(1.5)$ & $0(0.0)$ & $26(12.9)$ \\
\hline
\end{tabular}




\begin{tabular}{|c|c|c|c|c|c|}
\hline & & Hospital & & & Total \\
\hline \multirow[t]{2}{*}{ Six-month survival } & survived up to 6 months after discharge & $85(68.0)$ & $61(93.8)$ & $4(100.0)$ & $150(77.3)$ \\
\hline & died within 6 months after discharge & $40(32.0)$ & $4(6.2)$ & $0(0.0)$ & $44(22.7)$ \\
\hline \multirow[t]{4}{*}{ Decisional capacity } & given & $75(56.4)$ & $21(31.3)$ & $2(50.0)$ & $98(48.0)$ \\
\hline & not given & $37(27.8)$ & $20(29.9)$ & $1(25.0)$ & $58(28.4)$ \\
\hline & fluctuating & $11(8.3)$ & $5(7.5)$ & $1(25.0)$ & $17(8.3)$ \\
\hline & unclear & $10(7.5)$ & $21(31.3)$ & $0(0.0)$ & $31(15.2)$ \\
\hline \multicolumn{2}{|l|}{ Advance directive } & $23(17.4)$ & $3(4.5)$ & $0(0.0)$ & $26(12.8)$ \\
\hline \multicolumn{2}{|c|}{ Substitute decision-maker } & $43(32.6)$ & $33(49.3)$ & $1(25.0)$ & $77(37.9)$ \\
\hline
\end{tabular}

CEC: clinical ethics consultation.

Table 5shows the main ethical issues discussed in the CEC, covering a wide range of topics. Overall, the most common ethical issues refer to life-sustaining treatment (including resuscitation and artificial nutrition and hydration) (19.6\%), followed by coercive measures (16.7\%), maternal-foetal conflicts (12.3\%), goals of care or risk-benefit assessment (8.3\%) and assisted reproductive technology (6.9\%). While in the USB the three most prevalent topics are lifesustaining treatment (27.8\%), maternal-foetal conflicts (18.8\%) and assisted reproductive technology (10.5\%), in the UPK, the top issues are coercive measures (41.8\%), goals of care or risk-benefit assessment (14.9\%) and child welfare endangerment (13.4\%). In both hospitals, the top three issues are followed by the issue patient behaviour, that is, how to deal with demanding, non-adherent, substance-abusing, aggressive or self-harming patients $(6.0 \%$ and $7.5 \%$ in the USB and UPK, respectively). In addition to the main ethical issue in each case, further issues were included in the analysis if they were explicitly documented (and discussed) as such. Most prevalent secondary ethical issues refer to life-sustaining treatment (11.3\%), admission or discharge planning (7.8\%) and a substitute decision-maker (5.9\%). 
Table 5

Main ethical issues in CEC

\begin{tabular}{|c|c|c|c|c|}
\hline \multirow[t]{3}{*}{ Main ethical issue* } & \multicolumn{3}{|l|}{ Hospital } & \multirow[t]{2}{*}{ Total } \\
\hline & USB & UPK & External & \\
\hline & $n=133(\%)$ & $n=67(\%)$ & $\mathrm{n}=4(\%)$ & $n=204(\%)$ \\
\hline Treatment Decision Making & $37(27.8)$ & $47(70.1)$ & $1(25.0)$ & 85 (41.7) \\
\hline Coercive measures & $6(4.5)$ & $28(41.8)$ & $0(0.0)$ & 34 (16.7) \\
\hline Goals of care or risk-benefit assessment & $7(5.3)$ & $10(14.9)$ & $0(0.0)$ & $17(8.3)$ \\
\hline Patient behaviour & $8(6.0)$ & $5(7.5)$ & $0(0.0)$ & $13(6.4)$ \\
\hline Pain or suffering & $4(3.0)$ & $0(0.0)$ & $1(25.0)$ & $5(2.5)$ \\
\hline Informed consent or shared decision-making & $4(3.0)$ & $1(1.5)$ & $0(0.0)$ & $5(2.5)$ \\
\hline Patient wishes & $4(3.0)$ & $0(0.0)$ & $0(0.0)$ & $4(2.0)$ \\
\hline Admission or discharge planning & $2(1.5)$ & $2(3.0)$ & $0(0.0)$ & $4(2.0)$ \\
\hline Organ or tissue transplants & $2(1.5)$ & $0(0.0)$ & $0(0.0)$ & $2(1.0)$ \\
\hline Communication & $0(0.0)$ & $1(1.5)$ & $0(0.0)$ & $1(0.5)$ \\
\hline End-of-Life Decisions & $38(28.6)$ & $4(6.0)$ & $1(25.0)$ & $43(21.0)$ \\
\hline Life-sustaining treatment ${ }^{1}$ & $37(27.8)$ & $3(4.5)$ & $0(0.0)$ & 40 (19.6) \\
\hline Assistance in dying & $1(0.8)$ & $1(1.5)$ & $1(25.0)$ & $3(1.5)$ \\
\hline Reproductive Decisions & $41(30.8)$ & $0(0.0)$ & $0(0.0)$ & $41(20.1)$ \\
\hline Maternal-foetal conflict & $25(18.8)$ & $0(0.0)$ & $0(0.0)$ & $25(12.3)$ \\
\hline Assisted reproductive technology ${ }^{2}$ & $14(10.5)$ & $0(0.0)$ & $0(0.0)$ & $14(6.9)$ \\
\hline Gestational surrogacy & $1(0.8)$ & $0(0.0)$ & $0(0.0)$ & $1(0.5)$ \\
\hline Sterilisation & $1(0.8)$ & $0(0.0)$ & $0(0.0)$ & $1(0.5)$ \\
\hline Professionalism and Organisational Issues & $8(6.0)$ & $11(16.4)$ & $1(25.0)$ & $20(9.8)$ \\
\hline Child welfare endangerment & $0(0.0)$ & $9(13.4)$ & $0(0.0)$ & $9(4.4)$ \\
\hline Care management & $4(3.0)$ & $0(0.0)$ & $0(0.0)$ & $4(2.0)$ \\
\hline Resource allocation & $3(2.3)$ & $0(0.0)$ & $0(0.0)$ & $3(1.5)$ \\
\hline Medical mistakes & $1(0.8)$ & $1(1.5)$ & $0(0.0)$ & $2(1.0)$ \\
\hline Conflict of interest & $0(0.0)$ & $0(0.0)$ & $1(25.0)$ & $1(0.5)$ \\
\hline Human resources & $0(0.0)$ & $1(1.5)$ & $0(0.0)$ & $1(0.5)$ \\
\hline Substitute Decision Making & $9(6.8)$ & $4(6.0)$ & $1(25.0)$ & $14(6.9)$ \\
\hline Advance directive or advance care planning & $3(2.3)$ & $2(3.0)$ & $0(0.0)$ & $5(2.5)$ \\
\hline Decisional capacity & $1(0.8)$ & $1(1.5)$ & $1(25.0)$ & $3(1.5)$ \\
\hline Presumed patient will & $3(2.3)$ & $0(0.0)$ & $0(0.0)$ & $3(1.5)$ \\
\hline Substitute decision-maker & $2(1.5)$ & $1(1.5)$ & $0(0.0)$ & $3(1.5)$ \\
\hline Other & $0(0.0)$ & $1(1.5)$ & $0(0.0)$ & $1(0.5)$ \\
\hline \multicolumn{5}{|l|}{ * Ordered by overall prevalence within the groups. } \\
\hline${ }^{1}$ Including resuscitation and artificial nutriti & r hydration. & & & \\
\hline
\end{tabular}

Evaluation and outcomes of the CECs are shown in Table 6. In almost all cases, the CEC participants reached a consensus (98.5\%), often one that did not exist before (94.1\%). According to the requestor's description of the further clinical course, the results of the CEC were implemented in all cases, except in the rare cases in which the patient was transferred or died. CECs were rated as helpful (95.0\%) or partly helpful (5.0\%) by all requestors. CEC was often assessed as helpful for the care team (79.8\%) and the requesting person (60.3\%) but less often for the patient (38.2\%). Interdisciplinarity ( $86.5 \%)$, ethical analysis (80.2\%), 
structured discussion (67.5\%) and shared decision-making (63.2\%) were perceived, more often than not, as helpful. More than half also found the clarification of further procedures (57.6\%) and legal foundations (54.0\%) helpful. The CEC was less often seen as helpful in achieving educational goals such as promoting ethical competence (36.1\%) or developing one's own attitude (21.3\%). In almost all cases, the report was rated as helpful (97.0\%) or partly helpful (1.5\%). According to the requestors' assessment, the CESS responded in a timely manner in all cases. The CEC was carried out on time in all cases, with one exception due to a delay in finding an appointment within the care team.

Table 6

Evaluation and outcomes of CEC

\begin{tabular}{|c|c|c|c|c|c|}
\hline & & \multicolumn{3}{|l|}{ Hospital } & \multirow[t]{2}{*}{ Total } \\
\hline & & USB & UPK & External & \\
\hline & & $\mathrm{n}(\%)$ & $\mathrm{n}(\%)$ & $\mathrm{n}(\%)$ & $\mathrm{n}(\%)$ \\
\hline \multirow{2}{*}{$\begin{array}{l}\text { Consensus } \\
(n=204)\end{array}$} & yes & $132(99.2)$ & $65(97.0)$ & $4(100.0)$ & $201(98.5)$ \\
\hline & no & $1(0.8)$ & $2(3.0)$ & $0(0.0)$ & $3(1.5)$ \\
\hline \multirow{2}{*}{$\begin{array}{l}\text { New consensus } \\
(n=204)\end{array}$} & yes & $125(94.0)$ & $63(94.0)$ & $4(100.0)$ & $192(94.1)$ \\
\hline & no & $8(6.0)$ & $4(6.0)$ & $0(0.0)$ & $12(5.9)$ \\
\hline \multirow{5}{*}{$\begin{array}{l}\text { CEC results implemented } \\
(n=143)\end{array}$} & yes & 77 (92.8) & $29(96.7)$ & $1(100.0)$ & $107(93.9)$ \\
\hline & patient discharged before & $4(4.8)$ & $1(3.3)$ & $0(0.0)$ & $5(4.4)$ \\
\hline & patient died before & $1(1.2)$ & $0(0.0)$ & $0(0.0)$ & $1(0.9)$ \\
\hline & in progress & $1(1.2)$ & $0(0.0)$ & $0(0.0)$ & $1(0.9)$ \\
\hline & not available & 20 & 9 & 0 & 29 \\
\hline \multirow{3}{*}{$\begin{array}{l}\text { Helpfulness CEC } \\
(n=143)\end{array}$} & yes & $97(97.0)$ & $35(89.7)$ & $1(100.0)$ & $133(95.0)$ \\
\hline & yes, partially & $3(3.0)$ & $4(10.3)$ & $0(0.0)$ & $7(5.0)$ \\
\hline & not available & 3 & 0 & 0 & 3 \\
\hline \multirow[t]{4}{*}{ Helpfulness CEC report $(n=143)$} & yes & $91(95.8)$ & $36(100.0)$ & $1(100.0)$ & $128(97.0)$ \\
\hline & yes, partially & $2(2.1)$ & $0(0.0)$ & $0(0.0)$ & $2(1.5)$ \\
\hline & no & $2(2.1)$ & $0(0.0)$ & $0(0.0)$ & $2(1.5)$ \\
\hline & not available & 8 & 3 & 0 & 11 \\
\hline \multirow{3}{*}{$\begin{array}{l}\text { CESS response quick enough? } \\
(\mathrm{n}=72)^{1}\end{array}$} & yes & $58(100.0)$ & $13(100.0)$ & $1(100.0)$ & $72(100.0)$ \\
\hline & no & $0(0.0)$ & $0(0.0)$ & $0(0.0)$ & $0(0.0)$ \\
\hline & not available & 0 & 0 & 0 & 0 \\
\hline \multirow{3}{*}{$\begin{array}{l}\text { CEC held within reasonable time? } \\
(\mathrm{n}=72)^{1}\end{array}$} & yes & $57(98.3)$ & $13(100.0)$ & $1(100.0)$ & $71(98.6)$ \\
\hline & no & $1(1.7)$ & $0(0.0)$ & $0(0.0)$ & $1(1.4)$ \\
\hline & not available & 0 & 0 & 0 & 0 \\
\hline
\end{tabular}

Table 7 shows the prevalence of risk factors for moral conflicts discussed in CECs. Listed are risk factors with a prevalence of $\geq 25 \%$ overall or in at least one medical specialty (emergency or intensive care, internal medicine, surgery, women's health, psychiatry or other).

Overall, there is a high prevalence ( $\geq 50 \%$ ) for risk factors vulnerability $(100 \%)$, absent or unclear hospital policies (97.1\%), inadequate institutional framework (shift work) (83.3\%), inadequate understanding between patient and HCPs (73.5\%), inadequate communication (66.2\%), multimorbidity (66.2\%), disagreement between patients and HCPs about care (58.8\%), non-male patient (58.3\%), multiple care teams (53.4\%) and mental illness (50.5\%). Additionally, in all specialties specific risk factors are highly prevalent $(\geq 50 \%)$. In emergency or intensive care, the highly prevalent risk factors are unclear or missing patient preferences or $A D(71.4 \%)$, lack of decisional capacity (71.4\%), end-of-life situation (57.1\%) and multiple readmissions (50.0\%). In internal medicine, the highly prevalent risk factors are multiple readmissions (72.2\%), lack of decisional capacity (61.1\%) and low socio-economic status (52.8\%). In psychiatry, they are CPR code unclear or missing (86.4\%), self-harm and harm to others (74.2\%), inadequate understanding of disease on the part of the patient (71.2\%), low socioeconomic status (68.2\%), involuntary treatment (65.2\%), uncertainty about decisional capacity (53.0\%) and unclear or missing patient preferences or $A D$ (53.0\%). In surgery, they are unclear or missing patient preferences or AD (63.3\%) and multiple readmissions (50.0\%). In women's health, they are migration background (56.8\%, and maternal-foetal conflict (54.5\%). 
From the top 20 ranked risk factors in the literature (without subcategories), ten were moderately (25-49\%) or highly ( $\geq 50 \%$ ) prevalent overall or in specific specialties. Risk factors with a low prevalence include disagreement among relatives about care (13.7\%), different values or world views (13.2\%), inadequate resources $(13.2 \%)$, lack of crucial information or evidence (12.3\%), unnecessary suffering (8.8\%), unclear treatment plan among relatives (4.9\%), fear and risk behaviour (defensive medicine) (3.4\%), poor ethical climate in the team (1.5\%) and overworked HCPs (1.5\%). Inadequate moral attitude was not reported in CEC documentation. On average, 18 risk factors are prevalent in each CEC ( $M=18.2(4-32))$. 
Prevalence of moral risk factors in CEC ( $\geq 25 \%)$

\begin{tabular}{|c|c|c|c|c|c|c|c|}
\hline \multirow[t]{3}{*}{ Risk factor* } & \multicolumn{6}{|l|}{ Medical specialty } & \multirow[t]{2}{*}{ Total } \\
\hline & $\begin{array}{l}\text { Emergency or intensive } \\
\text { care }\end{array}$ & $\begin{array}{l}\text { Internal } \\
\text { Medicine }\end{array}$ & Surgery & $\begin{array}{l}\text { Women's } \\
\text { Health }\end{array}$ & Psychiatry & Other & \\
\hline & $n=14(\%)$ & $\mathrm{n}=36(\%)$ & $\begin{array}{l}n=30 \\
(\%)\end{array}$ & $\mathrm{n}=\mathbf{4 4}(\%)$ & $n=66(\%)$ & $\begin{array}{l}n=14 \\
(\%)\end{array}$ & $\begin{array}{l}n=204 \\
(\%)\end{array}$ \\
\hline \multicolumn{8}{|l|}{ Risk area: patient } \\
\hline Vulnerability & $14(100.0)$ & $36(100.0)$ & $\begin{array}{l}30 \\
(100.0)\end{array}$ & $44(100.0)$ & $66(100.0)$ & $\begin{array}{l}14 \\
(100.0)\end{array}$ & $\begin{array}{l}204 \\
(100.0)\end{array}$ \\
\hline - Multimorbidity & $11(78.6)$ & $33(91.7)$ & $23(76.7)$ & $9(20.5)$ & $46(69.7)$ & $13(92.9)$ & $135(66.2)$ \\
\hline - Non-male & $8(57.1)$ & $12(33.3)$ & $12(40.0)$ & $43(97.7)$ & $37(56.1)$ & $7(50.0)$ & $119(58.3)$ \\
\hline - Mental illness & $6(42.9)$ & $16(44.4)$ & $8(26.7)$ & $4(9.1)$ & $63(95.5)$ & $6(42.9)$ & $103(50.5)$ \\
\hline - Low socio-economic status & $3(21.4)$ & $19(52.8)$ & $7(23.3)$ & $9(20.5)$ & $45(68.2)$ & $4(28.6)$ & $87(42.6)$ \\
\hline - Lack of decisional capacity & $10(71.4)$ & $22(61.1)$ & $12(40.0)$ & $2(4.5)$ & $26(39.4)$ & $4(28.6)$ & $76(37.3)$ \\
\hline - Migration background & $2(14.3)$ & $12(33.3)$ & $12(40.0)$ & $25(56.8)$ & $20(30.3)$ & $4(28.6)$ & $75(36.8)$ \\
\hline - Very young or old age & $5(35.7)$ & $11(30.6)$ & $10(33.3)$ & $2(4.5)$ & $17(25.8)$ & $6(42.9)$ & $51(25.0)$ \\
\hline - Drug dependence & $3(21.4)$ & $13(36.1)$ & $2(6.7)$ & $1(2.3)$ & $30(45.5)$ & $1(7.1)$ & $50(24.5)$ \\
\hline - Lack of education (patient) & $0(0.0)$ & $5(13.9)$ & $2(6.7)$ & $2(4.5)$ & $17(25.8)$ & $1(7.1)$ & $27(13.2)$ \\
\hline - Alone & $4(28.6)$ & $4(11.1)$ & $2(6.7)$ & $0(0.0)$ & $6(9.1)$ & $1(7.1)$ & $17(8.3)$ \\
\hline $\begin{array}{l}\text { Inadequate understanding PAT- } \\
\mathrm{HCP}\end{array}$ & $12(85.7)$ & $29(80.6)$ & $25(83.3)$ & $17(38.6)$ & $60(90.9)$ & $7(50.0)$ & $150(73.5)$ \\
\hline $\begin{array}{l}\text { Disagreement about care PAT- } \\
\text { HCP }\end{array}$ & $3(21.4)$ & $17(47.2)$ & $17(56.7)$ & $26(59.1)$ & $52(78.8)$ & $5(35.7)$ & $120(58.8)$ \\
\hline $\begin{array}{l}\text { Unclear or missing preferences / } \\
A D\end{array}$ & $10(71.4)$ & $22(61.1)$ & 19 (63.3) & $9(20.5)$ & $35(53.0)$ & $6(42.9)$ & $101(49.5)$ \\
\hline Challenging clinical situation & $4(28.6)$ & $14(38.9)$ & $8(26.7)$ & $24(54.5)$ & $43(65.2)$ & $2(14.3)$ & $95(46.6)$ \\
\hline - Involuntary treatment & $2(14.3)$ & $5(13.9)$ & $2(6.7)$ & $1(2.3)$ & $43(65.2)$ & $0(0.0)$ & $53(26.0)$ \\
\hline - Maternal-foetal conflict & $0(0.0)$ & $0(0.0)$ & $1(3.3)$ & $24(54.5)$ & $0(0.0)$ & $0(0.0)$ & $25(12.3)$ \\
\hline Multiple re-admissions & $7(50.0)$ & $26(72.2)$ & $15(50.0)$ & $9(20.5)$ & $29(43.9)$ & $6(42.9)$ & $92(45.1)$ \\
\hline $\begin{array}{l}\text { Inadequate disease } \\
\text { understanding }\end{array}$ & $1(7.1)$ & $16(44.4)$ & $10(33.3)$ & $14(31.8)$ & 47 (71.2) & $2(14.3)$ & $90(44.1)$ \\
\hline Unclear or missing CPR code & $4(28.6)$ & $1(2.8)$ & $8(26.7)$ & $7(15.9)$ & $57(86.4)$ & $4(28.6)$ & $81(39.7)$ \\
\hline Self-harm and harm to others & $3(21.4)$ & $13(36.1)$ & $6(20.0)$ & $2(4.5)$ & $49(74.2)$ & $1(7.1)$ & $74(36.3)$ \\
\hline End-of-life situation & $8(57.1)$ & $16(44.4)$ & $11(36.7)$ & $4(9.1)$ & $5(7.6)$ & $5(35.7)$ & $49(24.0)$ \\
\hline Profound neurological deficit & $4(28.6)$ & $13(36.1)$ & $5(16.7)$ & $1(2.3)$ & $6(9.1)$ & $1(7.1)$ & $30(14.7)$ \\
\hline Acute renal failure & $4(28.6)$ & $13(36.1)$ & $7(23.3)$ & $0(0.0)$ & $1(1.5)$ & $2(14.3)$ & $27(13.2)$ \\
\hline $\begin{array}{l}\text { Repeated life-sustaining } \\
\text { treatments }\end{array}$ & $5(35.7)$ & $10(27.8)$ & $1(3.3)$ & $1(2.3)$ & $4(6.1)$ & $1(7.1)$ & $22(10.8)$ \\
\hline Recurrent aspiration pneumonia & $3(21.4)$ & $10(27.8)$ & $4(13.3)$ & $0(0.0)$ & $3(4.5)$ & $0(0.0)$ & $20(9.9)$ \\
\hline Prolonged artificial respiration & $4(28.6)$ & $4(11.1)$ & $2(6.7)$ & $0(0.0)$ & $0(0.0)$ & $0(0.0)$ & $10(4.9)$ \\
\hline \multicolumn{8}{|l|}{ Risk area: family } \\
\hline $\begin{array}{l}\text { Disagreement about care REL- } \\
\text { HCP }\end{array}$ & $6(42.9)$ & $11(30.6)$ & $8(26.7)$ & $15(34.1)$ & $16(24.2)$ & $2(14.3)$ & $58(28.4)$ \\
\hline Infrequent or missing visits & $2(14.3)$ & $7(19.4)$ & $3(10.0)$ & $1(2.3)$ & $29(43.9)$ & $4(28.6)$ & $46(22.5)$ \\
\hline Multiple family members & $4(28.6)$ & $11(30.6)$ & $12(40.0)$ & $5(11.4)$ & $11(16.7)$ & $2(14.3)$ & $45(22.1)$ \\
\hline
\end{tabular}

* Ordered by overall prevalence within the groups.

AD: advance directive; HCP: health care professionals; PAT: patient; REL: relatives. 


\begin{tabular}{|c|c|c|c|c|c|c|c|}
\hline \multirow[t]{3}{*}{ Risk factor* } & \multicolumn{6}{|l|}{ Medical specialty } & \multirow[t]{2}{*}{ Total } \\
\hline & $\begin{array}{l}\text { Emergency or intensive } \\
\text { care }\end{array}$ & $\begin{array}{l}\text { Internal } \\
\text { Medicine }\end{array}$ & Surgery & $\begin{array}{l}\text { Women's } \\
\text { Health }\end{array}$ & Psychiatry & Other & \\
\hline & $n=14(\%)$ & $n=36(\%)$ & $\begin{array}{l}n=30 \\
(\%)\end{array}$ & $n=44(\%)$ & $n=66(\%)$ & $\begin{array}{l}n=14 \\
(\%)\end{array}$ & $\begin{array}{l}n=204 \\
(\%)\end{array}$ \\
\hline $\begin{array}{l}\text { Inadequate disease } \\
\text { understanding }\end{array}$ & $5(35.7)$ & $8(22.2)$ & $5(16.7)$ & $10(22.7)$ & $10(15.2)$ & $1(7.1)$ & $39(19.1)$ \\
\hline Substitute function not fulfilled & $6(42.9)$ & $10(27.8)$ & $9(30.0)$ & $1(2.3)$ & $11(16.7)$ & $1(7.1)$ & $38(18.6)$ \\
\hline $\begin{array}{l}\text { Substitute missing or not } \\
\text { accessible }\end{array}$ & $4(28.6)$ & $8(22.2)$ & $3(10.0)$ & $0(0.0)$ & $14(21.2)$ & $0(0.0)$ & $29(14.2)$ \\
\hline \multicolumn{8}{|l|}{ Risk area: health care professional } \\
\hline Uncertainty about capacity & $6(42.9)$ & $17(47.2)$ & $5(16.7)$ & $4(9.1)$ & $35(53.0)$ & $4(28.6)$ & $71(34.8)$ \\
\hline Disagreement about care HCP & $2(14.3)$ & $13(36.1)$ & $8(26.7)$ & $7(15.9)$ & $25(37.9)$ & $3(21.4)$ & $58(28.4)$ \\
\hline Incoherent treatment plan & $2(14.3)$ & $14(38.9)$ & $12(40.0)$ & $4(9.1)$ & $9(13.6)$ & $3(21.4)$ & $44(21.6)$ \\
\hline Insufficient understanding HCP & $2(14.3)$ & $12(33.3)$ & $6(20.0)$ & $6(13.6)$ & $14(21.2)$ & $2(14.3)$ & $42(20.6)$ \\
\hline \multicolumn{8}{|l|}{ Risk area: system } \\
\hline $\begin{array}{l}\text { Absent or unclear hospital } \\
\text { policies }\end{array}$ & $13(92.9)$ & $36(100.0)$ & $27(90.0)$ & $44(100.0)$ & $65(98.5)$ & $13(92.9)$ & $198(97.1)$ \\
\hline $\begin{array}{l}\text { Inadequate institutional } \\
\text { framework }\end{array}$ & $14(100.0)$ & $35(97.2)$ & $\begin{array}{l}30 \\
(100.0)\end{array}$ & $29(65.9)$ & $52(78.8)$ & $10(71.4)$ & 170 (83.3) \\
\hline - Shift work & $14(100.0)$ & 35 (97.2) & $\begin{array}{l}30 \\
(100.0)\end{array}$ & $29(65.9)$ & $52(78.8)$ & $10(71.4)$ & 170 (83.3) \\
\hline Inadequate communication & $8(57.1)$ & $25(69.4)$ & $21(70.0)$ & $15(34.1)$ & $57(86.4)$ & $9(64.3)$ & $135(66.2)$ \\
\hline Multiple care teams & $9(64.3)$ & $24(66.7)$ & $19(63.3)$ & $30(68.2)$ & $22(33.3)$ & $5(35.7)$ & $109(53.4)$ \\
\hline
\end{tabular}

\section{Discussion}

\section{Characteristics, ethical issues and outcomes of CEC}

The institutional setting of this study, with an integrated CESS responsible for both a somatic and a psychiatric university hospital, is unusual and explains some of the results. It allows for a comparison between somatic and psychiatric CECs provided using the same approach. Thus, the study sheds light on the specific characteristics of CEC in psychiatry, which is still poorly studied (59-61). To enable comparison with international studies, results on USB-CECs will be discussed separately first.

Overall, the formal characteristics and demographic and clinical features of the patient population, as well as the main ethical issues of USB-CECs, are comparable to those of corresponding studies in the US $(35-39,41,43,44)$. However, there are some striking differences. A comparatively large share of CEC requests in the USB come from the women's health clinic and, accordingly, deal with ethical issues in maternal-foetal conflicts and assisted reproductive technologies. In the US studies, these topics rarely occur in $\operatorname{CEC~}(36,39,43)$ or are not separately reported $(35,37,38,41,44)$. According to Swiss law, to obtain a late abortion (after the 12th week of pregnancy), a pregnant woman who is not somatically at serious risk must claim severe mental distress comprehensible to the physician. The concept of severe mental distress allows considerable scope for interpretation and confronts HCPs with difficult decisions that are prepared for in CECs. Preimplantation genetic testing legally presupposes the risk of an embryo with a genetic predisposition for a severe disease. This raises the normative question of what is meant by "severe disease". In Switzerland, moreover, providers are legally obliged to prove to the health authorities that an individual ethical assessment was made, which is ensured by CEC at the USB. An in-depth analysis of this issue has been carried out by Muggli et al. (62).

Another distinctive feature is that relatively few requests for USB-CEC come from the ICU, compared to $32-55 \%$ in the US studies reviewed. Accordingly, the issue of life-sustaining treatment is not as prevalent as in many of those studies (35-38). This probably also explains why the prognosis, survival rate and decisional capacity of patients in USB-CECs are better on average than in the US studies $(35,41)$. Low numbers of ICU requests can be attributed to the fact that at the ICU ward, weekly team-internal ethical case discussions have been established in close collaboration with the CESS as a preventive approach to ethical decision-making. Most moral questions can be adequately answered within these case discussions - only particularly complex or burdensome cases lead to a CEC (29).

The issue of coercive measures, such as commitment, restraint or treatment against the patient's will or resistance, is no longer as prevalent in USB-CECs as it was in a previous study (40). However, it still seems to arise more often than in the US; none of the reviewed studies lists coercion as a separate ethical issue. 
In Switzerland, coercive measures are explicitly regulated at the federal level for mentally ill persons but not for patients in acute somatic hospitals, which explains the need for normative orientation. Coercive measures always involve a conflict of principles requiring careful ethical consideration, as in a CEC. An in-depth analysis of this issue in our CECs can be found in Montaguti et al. (63).

Although the proportion of patients with low socio-economic status (i.e. dependent on social welfare) or migration background in our study is quite high (over a third each), almost none are uninsured. In contrast, Robinson et al. report that more than one fifth of patients in CECs are un- or underinsured, significantly more than non-CEC patients (41). This difference can be attributed to the inclusive health insurance law in Switzerland and explains why ethical questions about access to health care are rarely addressed in our CECs. Surprisingly, this issue does not seem to play a more prominent role in CECs in the US either, although CEC could contribute to clarifying such issues (64-66).

Looking more closely at CEC in the UPK, half as many CECs were requested as in the USB, which roughly corresponds to the ratio of beds in the two institutions. It can therefore be assumed that there is a similar demand for CEC in psychiatry and in the somatic specialties. Not surprisingly, patients in UPKCECs suffer mainly from mental disorders; terminal illness (leading to death in a short time) is rare. Prognosis and decisional capacity are comparatively often unclear in these cases, and advance directives are almost never available. Although every second patient has a substitute decision-maker, he/she is not authorised to make decisions regarding the treatment of mental disorders in the event of incapacity. According to Swiss law, substitute decision-makers may only consent to or refuse treatment for somatic disorders. In the USB, family members are legally assigned the role of substitute decision-makers in the event of incapacity, provided there is no advance directive. In the UPK, on the other hand, the attending physician must decide on psychiatric treatment in the event of incapacity - even against the patient's wishes, where appropriate. Accordingly, decision-making in psychiatry often is complicated by uncertainty regarding prognosis and decisional capacity and, at the same time, related to a greater decision-making responsibility of the physician.

Regarding the main ethical issues discussed, institution-specific themes become visible. The three most prevalent ethical issues in USB-CECs - life-sustaining treatment, maternal-foetal conflicts and assisted reproductive technology - arise in relation to the specialised services offered by the USB; they do not (or only rarely) arise in the UPK. The high prevalence of coercive measures in UPK-CECs is hardly surprising in view of previous studies on ethical issues in psychiatry $(60,63,67)$. Other issues arising comparatively frequently are goals of care or risk-benefit assessment relating to controversial or potentially harmful treatments such as anti-androgenic treatment of sex offenders or medication-assisted treatment for patients with opioid dependence. Ethical questions on child welfare endangerment specific to child and adolescent psychiatry - for example, whether a child's well-being is in such danger that action (e.g. reporting to the authorities) is ethically justified or even required - have not yet been reported in studies on CEC.

The outcome variables examined show very good results in terms of reaching consensus among participants in CEC, implementation of CEC results, user experience (helpfulness) with CEC and response time of CESS in both hospitals. With regard to reaching consensus and helpfulness, the results are above average in international comparison $(20,21)$. To the authors' knowledge, the variables implementation and response time are investigated quantitatively here for the first time. Overall, the results indicate a high quality of CECs provided in both hospitals.

\section{Moral risk factors}

The scoping review reveals a wide range of moral risk factors in all dimensions relevant to clinical care: patient, family, providers, system. All four areas contain risk factors that are particularly frequent, according to the studies reviewed. Many of the common risk factors are also known as factors for moral distress in HCPs, which is an indication of their potency in clinical practice (68). Our earlier study gives some evidence that the most frequent risk factors are also potent (34): The 13 factors rated as moderately to strongly effective in the earlier study are also among the 20 most prevalent risk factors identified in the present review. These 13 factors are inadequate communication, disagreement among HCPs about care, disagreement between relatives and HCPs about care, vulnerability, different values or world views, inadequate understanding of disease on the part of relatives, incoherent treatment plan, lack of crucial information or evidence, disagreement among relatives about care, unclear treatment plan among relatives, unnecessary suffering, unclear or missing preferences or advance directive, end-of-life situation. These may, thus, be described as critical risk factors that are very likely to trigger moral problems.

Not all moral problems lead to CECs. The risk factors prevalent in CEC are only a subset of the factors that trigger moral problems. Half of the top 20 ranked moral risk factors in the literature are also moderately to highly prevalent in CECs. All patients in CECs are vulnerable and therefore at risk for undertreatment (69). The patients particularly often are multimorbid, non-male, mentally ill, lack decisional capacity or have a low socio-economic status. As with all patientrelated risk factors, there are considerable differences between the individual medical specialities, as they care for different patient populations. Lack of decisional capacity is a particularly potent vulnerability risk factor according to our previous study (34). It immediately raises ethical questions regarding, for example, the validity of advance directives, the proper role of the substitute decision-maker, or the presumed will or best interest of the patient.

In contrast, the highly prevalent risk factor of shift work of the HCPs seems to be of little significance as it applies to all inpatient situations in the hospital. However, excessive shift work has been shown to have a negative impact on quality of care, patient safety and general health of HCPs (70). It may therefore play a role as an indirect risk factor for moral problems. In our sample, however, there is little evidence of excessive shift work, especially since related factors such as inadequate resources, overworked HCPs or lack of management support are not highly prevalent.

Inadequate communication in general and inadequate understanding between patient and HCPs are highly prevalent risk factors documented in our CECs. Absent, incomplete, incomprehensible, inaccurate or insensitive communication is well known as a reason for conflicts between HCPs, patients and relatives $(71,72)$. Inadequate communication may be morally significant in several ways, either by infringing patient rights or professional standards or by concealing, delaying or wrongly suggesting value conflicts. Inadequate understanding between patient and HCPs, often the consequence of inadequate communication, prevents key ethical standards such as informed consent, shared decision-making or advance care planning, from being met. Inadequate communication is, according to our previous study, the most potent risk factor, making it a highly prevalent and potent factor for moral problems leading to CECs. 
The risk factor disagreement between patient and HCPs about care is also highly prevalent and presumably also potent. Persistent disagreement between patient and HCPs inevitably leads to moral conflicts unless one party withdraws from the situation. In CECs in emergency and intensive care, this risk factor is less prevalent, as many of these patients are no longer capable of communication. Accordingly, disagreement between relatives and HCPs and other familyrelated risk factors are more prevalent in these situations and are just as likely to lead to moral conflicts. Disagreements between relatives and HCPs were found, in our previous study, to be highly potent, as were disagreements among HCPs (34). Persistent disagreement about care thus seems to be a highly prevalent and potent factor for moral problems leading to CEC. Figure 2 presents an overview of highly prevalent and (according to our previous study) potent risk factors in the various medical specialties.

Also highly prevalent is the risk factor absent or lacking hospital policies, which is not recognised in the literature as a common factor. Regarding the ethical issues in our CECs, there are hardly any specific institutional policies that could have been consulted. Although there are ethics guidelines on various topics available at the national level, they are often not specific enough to be considered helpful in individual cases. During the observation period, institutional policies have been developed by the CESS on ethically relevant topics such as CPR decisions, assisted suicide or patient violence. Developing such policies is considered to be a useful measure to prevent moral conflicts (34).

Some common risk factors identified in the literature, such as different values and world views, inadequate resources, inadequate moral attitude and unnecessary suffering, are less prevalent in our CECs. This is probably due to several reasons. There may be methodological reasons in the case of different values and world views and inadequate moral attitude. These were coded only if the documentation explicitly referred to different social, cultural, religious or metaphysical value contexts or inadequate moral attitudes such as lack of moral sensitivity, commitment or fairness. Due to the high proportion of patients with a migration background (36.8\%), it can be assumed that different value contexts were de facto more prevalent than indicated (13.2\%). Inadequate moral attitudes are probably not documented because CEC usually does not include an ethical assessment of character. Inadequate resources such as time, money, personnel or services are documented in $13.2 \%$ of our CECs. Rationing in health care, or withholding medically indicated services for reasons of cost or scarcity, has been reported to a considerable extent for Switzerland $(69,73)$. However, minor cases of inadequate resources or rationing in everyday clinical practice may not lead to requests for CEC. Unnecessary suffering, that is suffering that could be successfully treated, is problematic from an ethical perspective, assuming that every patient has a moral right to appropriate symptom control (74). Undertreatment of pain still is a problem in Western European hospitals: about $10 \%$ of postsurgical patients in Germany and Austria retrospectively report having desired more pain treatment than they received (75). Pain and palliative care services can probably deal with such cases successfully if they are involved. CEC may only be requested in cases of greater moral complexity, such as when substitute decision-makers refuse analgesics or patients are suffering from opioid dependency.

\section{Limitations}

The results of our study are limited regarding validity and generalisability due to the absence of a control group and the specific setting of the study. Also, the number of cases in the different medical specialities is relatively small and does not allow statistical analysis on different risk profiles. The results are therefore primarily suitable for generating reasonable hypotheses about moral risk factors that need to be tested by further research. Case series are susceptible to several biases, especially selection bias and measurement bias. Since all sufficiently documented case-related CECs were included in the series, selection bias seems to be negligible. However, it must be kept in mind that only a - presumably small - proportion of moral problems in patient care leads to CEC. Since the request for CEC depends on various parameters independent of the moral problem, including availability and range of CES, knowledge and attitude of HCPs about CES, and the moral sensibility, competence or resilience of HCPs, it cannot be presupposed that CECs accurately reflect the content and prevalence of moral problems in the institution. The risk of measurement bias is reduced, as this study uses highly standardised report templates and feedback forms. However, some moral risk factors from the literature have not been systematically captured and are difficult to operationalise. This may have led to an underestimation of the prevalence of these risk factors.

\section{Conclusions}

Our study shows that CEC is useful in both somatic medicine and psychiatry to find consensual and helpful solutions to moral conflicts. While the need for CEC in psychiatry is proportionally similar to that in somatic medicine, the thematic focus is different. In psychiatry, ethical questions about coercive measures, risk and benefit assessments of potentially harmful side effects of psychiatric treatments, and child welfare endangerment are most prevalent in CEC. Ethical decision-making tends to be complex due to greater uncertainty about the patient's prognosis and decision-making capacity and greater decisionmaking responsibility on the part of the physician. In the USB, ethical issues in maternal-foetal conflicts and in relation to assisted reproductive technologies are comparatively often discussed in CEC, alongside decisions on life-sustaining measures and a variety of other issues, most of them familiar from other studies. Effective preventive interventions, such as the weekly team-internal ethical case discussions on the USB ICU ward, may lead to an increase of CES, including the team-internal approach, but a decrease in the request for formal CEC.

There is a wide range of moral risk factors in all dimensions relevant to clinical care. Regarding the patient, patient vulnerability, end-of-life situations, unnecessary suffering (due to suboptimal pain treatment) and unclear or missing information about patient preferences or advance directives are the most prevalent risk factors. In relation to patients' families, it is a lack of understanding of the disease, disagreement with HCPs or each other, and unclear treatment plans. Concerning HCPs, incoherent treatment plans, inadequate moral attitude, disagreement with each other and overwork are the most common factors promoting moral conflicts. Systemic risk factors include inadequate communication, divergent values or world views among those involved, inadequate resources and lack of crucial information or evidence. Most of these moral risk factors can be found in cases discussed in CEC. As not all moral problems lead to $\mathrm{CEC}$, not all risk factors are as prevalent as the literature suggests. Highly prevalent and - according to our previous study - potent in CEC are the following risk factors: vulnerability, inadequate understanding between patient and HCP, inadequate communication and disagreement about care between patients and HCPs. Our analysis reveals that almost all risk factors are specific in that they are not equally prevalent or - presumably - potent in all medical specialities. We have presented a preliminary overview of the risk factors specific to various medical specialties. Further empirical research is needed to validate our results

Page 17/23 
and create different discipline-specific risk profiles. Such risk profiles will enhance the development of targeted and efficient preventive interventions to address moral problems in patient care early on.

\section{Abbreviations}

CEC

Clinical ethics consultation

CES

Clinical ethics support

CESS

Clinical ethics support service

$\mathrm{HCP}$

Health care professional

PCES

Preventive clinical ethics support

UPK

University Psychiatric Clinics Basel

USB

University Hospital Basel

\section{Declarations}

\section{Ethics approval and consent to participate}

The Ethics Committee Northwest and Central Switzerland (EKNZ) waived the need for ethics approval and consent to participate for this study, as it does not fall within the scope of the Swiss Human Research Act (Req-2021-00931). All methods were carried out in accordance with relevant guidelines and regulations.

\section{Consent for publication}

Not applicable.

\section{Availability of data and materials}

The datasets generated and/or analysed during the current study are not publicly available due to confidentiality reasons but are available from the corresponding author on reasonable request.

\section{Competing interests}

The authors declare that they have no competing interests.

\section{Funding}

This research received no specific grant from any funding agency in the public, commercial, or not-for-profit sectors.

\section{Authors' contributions}

JS conceptualised the study, carried out the analysis, interpreted its results, wrote the first draft and revised the manuscript. SRT supervised the study, critically revised the first draft and supported the interpretation of the results. All authors read and approved the final manuscript.

\section{Acknowledgements}

The authors thank Klaus Schmeck and Anna Westermair for their helpful comments on previous versions of this manuscript.

\section{Authors' information}

Jan Schürmann is a clinical ethicist at the Clinical Ethics Unit of the University Hospital Basel and the University Psychiatric Clinics Basel.

Stella Reiter-Theil is professor emeritus of medical and health ethics and former director of the Clinical Ethics Unit of the University Hospital Basel and the University Psychiatric Clinics Basel.

\section{References}

1. Fox E, Danis M, Tarzian AJ, Duke CC. Ethics Consultation in U.S. Hospitals: A National Follow-Up Study. Am J Bioeth 2021:1-14.

2. Schochow M, Schnell D, Steger F. Implementation of Clinical Ethics Consultation in German Hospitals. Sci Eng Ethics 2019; 25(4):985-91. 
3. Slowther AM, McClimans L, Price C. Development of clinical ethics services in the UK: a national survey. J Med Ethics 2012; 38(4):210-4. Available from: URL: https://jme.bmj.com/content/38/4/210.

4. Ackermann S, Balsiger L, Salathé M. Ethikstrukturen an Akutspitälern, Psychiatrischen Kliniken und Rehabilitationskliniken der Schweiz. Bioethica Forum 2016; 9(2):52-9.

5. Swiss Academy of Medical Sciences. Ethics support in medicine. Bern; 2017.

6. Zentrale Ethikkommission der Bundesärztekammer. Stellungnahme: Ethikberatung in der klinischen Medizin. Berlin: Zentrale Ethikkommission der Bundesärztekammer; 2006.

7. Akademie für Ethik in der Medizin. Standards für Ethikberatung in Einrichtungen des Gesundheitswesens. Ethik Med 2010; 22(2):149-53.

8. American Society for Bioethics and Humanities. Core Competencies for Healthcare Ethics Consultation. Glenview.

9. American Society for Bioethics and Humanities. Code of Ethics and Professional Responsibilites for Healthcare Ethics Consultants. Chicago: American Society for Bioethics and Humanities; 2014.

10. Dörries A, Simon A, Neitzke G, Vollmann J. Implementing clinical ethics in German hospitals: content, didactics and evaluation of a nationwide postgraduate training programme. Journal of medical ethics 2010; 36(12):721-6.

11. American Society for Bioethics and Humanities. Improving competencies in clinical ethics consultation: An education guide. Chicago.

12. Swiss Academy of Medical Sciences. Ethics training for health professionals. Bern; 2019.

13. Berkowitz KA, Katz AL, Powderly KE, Spike JP. Quality Assessment of the Ethics Consultation Service at the Organizational Level: Accrediting Ethics Consultation Services. The American journal of bioethics: AJOB 2016; 16(3):42-4.

14. Tozzo P, Mazzi A, Aprile A, Rodriguez D, Caenazzo L. Certification ISO 9001 in clinical ethics consultation for improving quality and safety in healthcare. International journal for quality in health care: journal of the International Society for Quality in Health Care 2018; 30(6):486-91.

15. Bruce CR, Feudtner C, Davis D, Benner MB. Developing, Administering, and Scoring the Healthcare Ethics Consultant Certification Examination. Hastings Cent Rep 2019; 49(5):15-22.

16. Fins JJ, Kodish E, Cohn F, Danis M, Derse AR, Dubler NN et al. A Pilot Evaluation of Portfolios for Quality Attestation of Clinical Ethics Consultants. The American journal of bioethics: AJOB 2016; 16(3):15-24.

17. Pearlman RA, Foglia MB, Fox E, Cohen JH, Chanko BL, Berkowitz KA. Ethics Consultation Quality Assessment Tool: A Novel Method for Assessing the Quality of Ethics Case Consultations Based on Written Records. The American journal of bioethics: AJOB 2016; 16(3):3-14.

18. Wasson K, Parsi K, McCarthy M, Siddall VJ, Kuczewski M. Developing an Evaluation Tool for Assessing Clinical Ethics Consultation Skills in Simulation Based Education: The ACES Project. HEC forum: an interdisciplinary journal on hospitals' ethical and legal issues 2016; 28(2):103-13.

19. Yoon NYS, Ong YT, Yap HW, Tay KT, Lim EG, Cheong CWS et al. Evaluating assessment tools of the quality of clinical ethics consultations: a systematic scoping review from 1992 to 2019. BMC Medical Ethics 2020; 21(1):51. Available from: URL:

https://bmcmedethics.biomedcentral.com/articles/10.1186/s12910-020-00492-4.

20. Au SS, Couillard P, Des Roze Ordons A, Fiest KM, Lorenzetti DL, Jette N. Outcomes of Ethics Consultations in Adult ICUs: A Systematic Review and MetaAnalysis. Critical care medicine 2018; 46(5):799-808.

21. Haltaufderheide J, Nadolny S, Gysels M, Bausewein C, Vollmann J, Schildmann J. Outcomes of clinical ethics support near the end of life: A systematic review. Nurs Ethics 2020:969733019878840-969733019878840.

22. Schildmann J, Nadolny S, Haltaufderheide J, Gysels M, Vollmann J, Bausewein C. Ethical case interventions for adult patients. Cochrane Database of Systematic Reviews 2019; (7).

23. Chervenak FA, McCullough LB. Clinical guides to preventing ethical conflicts between pregnant women and their physicians. American Journal of Obstetrics and Gynecology 1990; 162(2):303-7.

24. Forrow L, Arnold RM, Parker LS. Preventive ethics: expanding the horizons of clinical ethics. The Journal of clinical ethics 1993; 4(4):287-94.

25. Frolic A, Jennings B, Seidlitz W, Andreychuk S, Djuric-Paulin A, Flaherty B et al. From reactive to proactive: developing a valid clinical ethics needs assessment survey to support ethics program strategic planning (part 1 of 2). HEC forum: an interdisciplinary journal on hospitals' ethical and legal issues 2013; 25(1):47-60.

26. Pavlish CL, Henriksen J, Brown-Saltzman K, Robinson EM, Warda US, Farra C et al. A Team-Based Early Action Protocol to Address Ethical Concerns in the Intensive Care Unit. American Journal of Critical Care 2020 [cited 2020 Jan 14]; 29(1):49-61.

27. Schürmann J, Mühleck M, Perler C, Schmeck K, Reiter-Theil S. Ethische Herausforderungen in der forensischen Kinder- und Jugendpsychiatrie. Eine qualitative Beobachtungsstudie und ein Instrument zur Früherkennung und Frühintervention. Ethik Med 2021; 33(1):31-49.

28. Schmitz D, Groß D, Frierson C, Schubert GA, Schulze-Steinen H, Kersten A. Ethics rounds: affecting ethics quality at all organisational levels. J. Med. Ethics 2018; 44(12):805-9.

29. Meyer-Zehnder B, Barandun Schäfer U, Wesch C, Reiter-Theil S, Pargger H. Weekly internal ethical case discussions in an intensive care unit - Results based on 9 years of experience with a highly structured approach. Critical Care Explorations 2021; 3(3):1-9.

30. Dowdy MD, Robertson C, Bander JA. A study of proactive ethics consultation for critically and terminally ill patients with extended lengths of stay. Critical care medicine 1998; 26(2):252-9.

31. Andereck WS, McGaughey JW, Schneiderman LJ, Jonsen AR. Seeking to reduce nonbeneficial treatment in the ICU: an exploratory trial of proactive ethics intervention*. Critical care medicine 2014; 42(4):824-30. 
32. McCullough LB. The Professional Medical Ethics Model of Decision Making Under Conditions of Clinical Uncertainty. Medical Care Research and Review 2013; 70(1_suppl):141S-158S.

33. National Center for Ethics in Health Care. Preventive Ethics. Addressing Ethics Quality Gaps on a Systems Level. 2nd ed. Washington, DC: U.S. Department of Veteran Affairs; 2015.

34. Schürmann J, Vaitaityte G, Reiter-Theil S. Preventing moral conflicts in patient care: Insights from a mixed-methods study with clinical experts. Clinical Ethics 2021:147775092110572.

35. Swetz KM, Crowley ME, Hook C, Mueller PS. Report of 255 clinical ethics consultations and review of the literature. Mayo Clinic Proceedings 2007; 82(6):686-91. Available from: URL: https://pubmed.ncbi.nlm.nih.gov/17550748/.

36. Tapper EB, Vercler CJ, Cruze D, Sexson W. Ethics consultation at a large urban public teaching hospital. Mayo Clinic proceedings 2010; 85(5):433-8.

37. Bruce CR, Smith ML, Hizlan S, Sharp RR. A systematic review of activities at a high-volume ethics consultation service. The Journal of clinical ethics 2011; 22(2):151-64.

38. Moeller JR, Albanese TH, Garchar K, Aultman JM, Radwany S, Frate D. Functions and outcomes of a clinical medical ethics committee: a review of 100 consults. HEC forum: an interdisciplinary journal on hospitals' ethical and legal issues 2012; 24(2):99-114.

39. Wasson K, Anderson E, Hagstrom E, McCarthy M, Parsi K, Kuczewski M. What Ethical Issues Really Arise in Practice at an Academic Medical Center? A Quantitative and Qualitative Analysis of Clinical Ethics Consultations from 2008 to 2013. HEC forum: an interdisciplinary journal on hospitals' ethical and legal issues 2016; 28(3):217-28.

40. Reiter-Theil S, Schürmann J. The 'Big Five' in 100 Clinical Ethics Consultation Cases. Evaluating three years of ethics support in the Basel University Hospitals. Bioethica Forum 2016; 9(2):12-22.

41. Robinson EM, Cage W, Erler K, Brackett S, Bandini J, Cist A et al. Structure, Operation, and Experience of Clinical Ethics Consultation 2007-2013: A Report from the Massachusetts General Hospital Optimum Care Committee. The Journal of clinical ethics 2017; 28(2):137-52.

42. Corbett V, Epstein AS, McCabe MS. Characteristics and Outcomes of Ethics Consultations on a Comprehensive Cancer Center's Gastrointestinal Medical Oncology Service. HEC forum: an interdisciplinary journal on hospitals' ethical and legal issues 2018; 30(4):379-87.

43. Milliken A, Courtwright A, Grace P, Eagan-Bengston E, Visser M, Jurchak M. Ethics Consultations at a Major Academic Medical Center: A Retrospective, Longitudinal Analysis. AJOB empirical bioethics 2020; 11(4):275-86.

44. Harris KW, Cunningham TV, Hester DM, Armstrong K, Kim A, Harrell FE et al. Comparison Is Not a Zero-Sum Game: Exploring Advanced Measures of Healthcare Ethics Consultation. AJOB empirical bioethics 2021; 12(2):123-36.

45. Cummings $P$, Weiss NS. Case series and exposure series: the role of studies without controls in providing information about the etiology of injury or disease. Inj Prev 1998; 4(1):54-7. Available from: URL: https://injuryprevention.bmj.com/content/4/1/54.

46. Reiter-Theil S. Dealing with the normative dimension in clinical ethics consultation. Cambridge quarterly of healthcare ethics: CQ : the international journal of healthcare ethics committees 2009; 18(4):347-59.

47. Reiter-Theil S. Initiating and maintaining clinical ethics support in psychiatry. Ten tasks and challenges - And how to meet them. Clinical Ethics 2016 ; $11(2-3): 45-53$.

48. Tricco AC, Lillie E, Zarin W, O'Brien KK, Colquhoun H, Levac D et al. PRISMA Extension for Scoping Reviews (PRISMA-ScR): Checklist and Explanation. Ann Intern Med 2018; 169(7):467-73.

49. Munn Z, Barker T, Moola S, Tufanaru C, Stern C, McArthur A et al. Methodological quality of case series studies; 2020.

50. Mayring P. Qualitative content analysis: theoretical foundation, basic procedures and software solution. Klagenfurt; 2014.

51. Pavlish CL, Brown-Saltzman K, Robinson EM, Henriksen J, Warda US, Farra C et al. An Ethics Early Action Protocol to Promote Teamwork and Ethics Efficacy. Dimens Crit Care Nurs 2021; 40(4):226-36.

52. Pavlish C, Hellyer J, Brown-Saltzman K, Miers A, Squire K. Screening situations for risk of ethical conflicts: a pilot study. American journal of critical care: an official publication, American Association of Critical-Care Nurses 2015; 24(3):248-56.

53. Anderson-Shaw L, Ahrens W, Fetzer M. Ethics consultation in the emergency department. JONA'S healthcare law, ethics and regulation 2007; 9(1):32-5.

54. Epstein EG. Preventive ethics in the intensive care unit. AACN advanced critical care 2012; 23(2):217-24.

55. Heyl J. Early Indicators for Ethics Reviews. Health Care Ethics USA 2008; 16(3):2-4.

56. Morgenstern L. Proactive bioethics screening: a prelude to bioethics consultation. The Journal of clinical ethics 2005; 16(2):151-5.

57. Pavlish C, Brown-Saltzman K, Hersh M, Shirk M, Nudelman O. Early indicators and risk factors for ethical issues in clinical practice. Journal of nursing scholarship: an official publication of Sigma Theta Tau International Honor Society of Nursing 2011; 43(1):13-21.

58. Pavlish C, Brown-Saltzman K, Dirksen KM, Fine A. Physicians' Perspectives on Ethically Challenging Situations: Early Identification and Action. AJOB Empirical Bioethics 2015; 6(3):28-40.

59. Reiter-Theil S, Schurmann J, Schmeck K. [Clinical ethics in psychiatry: state of the art]. Psychiatrische Praxis 2014; 41(7):355-63.

60. Syse I, Førde R, Pedersen R. Clinical ethics committees - also for mental health care? The Norwegian experience. Clinical Ethics 2016; 11(2-3):81-6.

61. Haltaufderheide J, Gather J, Juckel G, Schildmann J, Vollmann J. Types of Ethical Problems and Expertise in Clinical Ethics Consultation in Psychiatry Insights From a Qualitative Empirical Ethics Study. Front. Psychiatry 2021; 12.

62. Muggli M, Geyter C de, Reiter-Theil S. Shall parent / patient wishes be fulfilled in any case? A series of 32 ethics consultations: from reproductive medicine to neonatology. BMC Medical Ethics 2019; 20(1):4. 
63. Montaguti E, Schürmann J, Wetterauer C, Picozzi M, Reiter-Theil S. Reflecting on the Reasons Pros and Cons Coercive Measures for Patients in Psychiatric and Somatic Care: The Role of Clinical Ethics Consultation. A Pilot Study. Frontiers in Psychiatry 2019; 10(441).

64. Strech D, Hurst S, Danis M. The role of ethics committees and ethics consultation in allocation decisions: a 4-stage process. Medical care 2010; 48(9):821-6.

65. Schürmann J, Meyer-Zehnder B, Mertz M, Albisser schleger H, Schlögl M, Kressig RW et al. Fairness and transparency in bedside micro-allocation: Improving the ethical competence of clinical staff. In: Danis M, Fleck L, Hurst SA, Forde R, Slowther A, editors. Fair Resource Allocation and Rationing at the Bedside. Oxford: Oxford University Press; 2014. p. 301-22.

66. Magelssen M, Bærøe K. Can clinical ethics committees be legitimate actors in bedside rationing? BMC Medical Ethics 2019; 20(1):97. Available from: URL: https://bmcmedethics.biomedcentral.com/articles/10.1186/s12910-019-0438-y.

67. Molewijk B, Engerdahl IS, Pedersen R. Two years of moral case deliberations on the use of coercion in mental health care: Which ethical challenges are being discussed by health care professionals? Clinical Ethics 2016.

68. Atashzadeh-Shoorideh F, Tayyar-Iravanlou F, Chashmi ZA, Abdi F, Cisic RS. Factors affecting moral distress in nurses working in intensive care units: A systematic review. Clinical Ethics 2021; 16(1):25-36.

69. Hurst S, Forde R, Reiter-Theil S, Slowther A-M, Perrier A, Pegoraro R et al. Physicians' views on resource availability and equity in four European health care systems. BMC Health Services Research 2007; 7(1):137.

70. Dall'Ora C, Ball J, Recio-Saucedo A, Griffiths P. Characteristics of shift work and their impact on employee performance and wellbeing: A literature review. International journal of nursing studies 2016; 57:12-27.

71. Azoulay E, Timsit JF, Sprung CL, Soares M, Rusinova K, Lafabrie A et al. Prevalence and factors of intensive care unit conflicts: the conflicus study. American journal of respiratory and critical care medicine 2009; 180(9):853-60.

72. Boyle DK, Miller PA, Forbes-Thompson SA. Communication and end-of-life care in the intensive care unit: patient, family, and clinician outcomes. Critical care nursing quarterly $2005 ; 28(4): 302-16$.

73. Schubert M, Ausserhofer D, Desmedt M, Schwendimann R, Lesaffre E, Li B et al. Levels and correlates of implicit rationing of nursing care in Swiss acute care hospitals-a cross sectional study. International journal of nursing studies 2013; 50(2):230-9.

74. Brennan F, Carr DB, Cousins M. Pain management: a fundamental human right. Anesthesia and analgesia 2007; 105(1):205-21.

75. Komann M, Baumbach P, Stamer UM, Weinmann C, Arnold C, Pogatzki-Zahn E et al. Desire to Receive More Pain Treatment - A Relevant Patient-Reported Outcome Measure to Assess Quality of Post-Operative Pain Management? Results From 79,996 Patients Enrolled in the Pain Registry QUIPS from 2016 to 2019. The Journal of Pain 2021; 22(6):730-8. Available from: URL: https://www.jpain.org/article/S1526-5900(21)00011-0/fulltext.

\section{Figures}




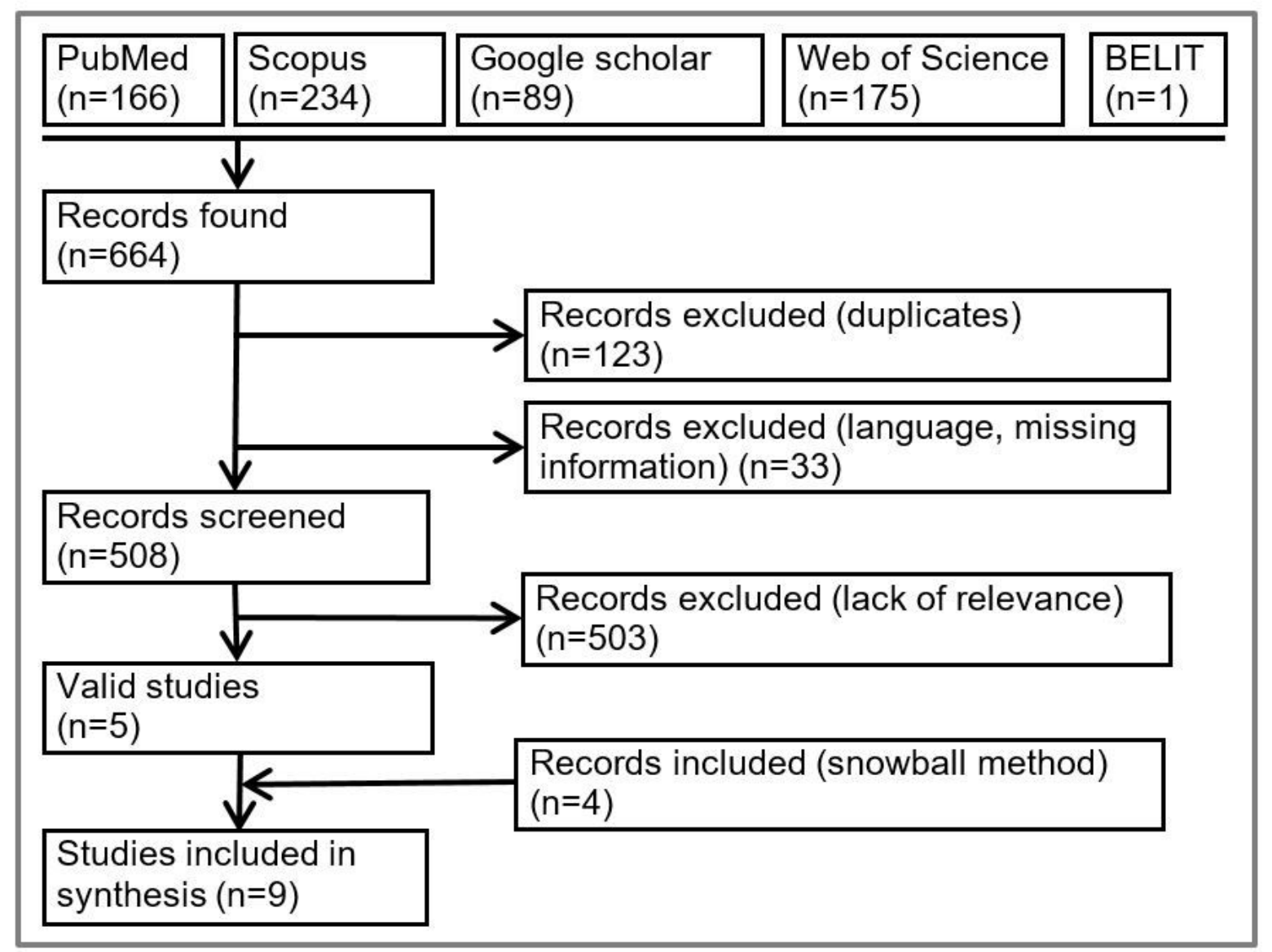

Figure 1

PRISMA flow diagram 


\section{Medical specialty}

\section{Emergency or intensive care}

\section{Internal medicine}

\section{Surgery}

Women's health

Psychiatry

Vulnerability
- Multimorbidity
- Lack of decisional
capacity
Inadequate under-
standing PAT-HCP
Unclear or missing
preferences / AD
End-of-life situation
Inadequate communi-
cation
Disagreement about
care REL-HCP
Substitute function not
fulfilled
Uncertainty about ca-
pacity

Vulnerability

- Multimorbidity

- Lack of decisional capacity

Inadequate understanding PAT-HCP

Inadequate communication

Unclear or missing preferences / AD

Disagreement about care PAT-HCP

Uncertainty about capacity

End-of-life situation Inadequate understanding of disease
Vulnerability

- Multimorbidity

- Lack of decisional capacity

Inadequate understanding PAT-HCP

Inadequate communication

Unclear or missing preferences / AD

Disagreement about care PAT-HCP

Incoherent treatment plan

End-of-life situation
Vulnerability

- Migration background

Disagreement about care PAT-HCP

Maternal-fetal conflict Inadequate understanding PAT-HCP

Disagreement about care REL-HCP

Inadequate communication
Vulnerability

- Multimorbidity

- Low socio-economic status

- Drug dependence

Inadequate understanding PAT-HCP Inadequate communication

Disagreement about care PAT-HCP

Self-harm and harm to others

Inadequate understanding of disease Involuntary treatment

Unclear or missing preferences / AD

\section{Figure 2}

\section{Highly prevalent and potent moral risk factors leading to CEC in different medical specialties.}

AD: advance directive; HCP: health care professionals; PAT: patient; REL: relatives. 